\title{
Lower Bounds for Two-Level Additive Schwarz Preconditioners with Small Overlap *
}

\author{
Susanne C. Brenner \\ Department of Mathematics \\ University of South Carolina \\ Columbia, SC 29208
}

Summary. Lower bounds for the condition numbers of the preconditioned systems are obtained for two-level additive Schwarz preconditioners for both second order and fourth order problems. They show that the known upper bounds are sharp in the case of a small overlap.

Mathematics Subject Classification (1991): 65N55, 65N30.

\section{Introduction}

Let $\Omega=(0,1) \times(0,1), V=H_{0}^{1}(\Omega)$ for the second order model problem and $H_{0}^{2}(\Omega)$ for the fourth order model problem, and the variational form $a(\cdot, \cdot)$ be defined by either

$$
a\left(v_{1}, v_{2}\right)=\int_{\Omega} \nabla v_{1} \cdot \nabla v_{2} d x \quad \forall v_{1}, v_{2} \in H_{0}^{1}(\Omega)
$$

for the second order case, or

$$
a\left(v_{1}, v_{2}\right)=\int_{\Omega} \sum_{i, j=1,2}\left(v_{1}\right)_{x_{i} x_{j}}\left(v_{2}\right)_{x_{i} x_{j}} d x \quad \forall v_{1}, v_{2} \in H_{0}^{2}(\Omega)
$$

for the fourth order case.

Consider the following variational problem:

Find $u \in V$ such that

$$
a(u, v)=\int_{\Omega} f v d x \quad \forall v \in V
$$

where $f \in L_{2}(\Omega)$.

The variational problem (1.3) can be discretized using the $P_{1}$ conforming finite element (cf. Figure 1) in the second order case and the Hsieh-Clough-Tocher macro element (cf. Figure 2 and [11]) in the fourth order case. The nodal variables of these elements are depicted in Figure 1 and Figure 2 according to the conventions in [10] and [6].

* This work was supported in part by the National Science Foundation under Grant No. DMS-96-00133. 


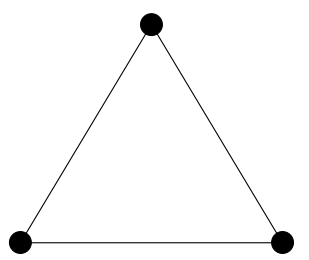

Figure 1.

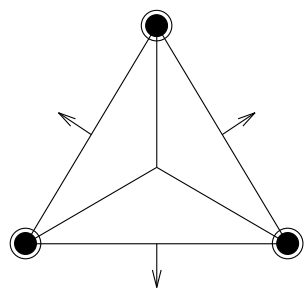

Figure 2.

Anticipating the use of two-level domain decomposition preconditioners, we construct a triangulation of $\Omega$ in the following way. Let $\Omega$ be divided into $J=2^{2 k}$ nonoverlapping squares $\widehat{\Omega}_{1}, \ldots, \widehat{\Omega}_{J}$ (cf. Figure 3 where $\mathrm{k}=2$ ). By adding a diagonal to each $\widehat{\Omega}_{j}$ we obtain a triangulation $\mathcal{T}_{H}$ of $\Omega$ (cf. Figure 4). Then we perform a dyadic subdivision of $\mathcal{T}_{H}$ to obtain the triangulation $\mathcal{T}_{h}$ (cf. Figure 5). Here $H$ and $h$ are the lengths of the horizontal edges in $\mathcal{T}_{H}$ and $\mathcal{T}_{h}$ respectively.

\begin{tabular}{|l|l|l|l|}
\hline$\widehat{\Omega}_{1}$ & $\widehat{\Omega}_{2}$ & $\widehat{\Omega}_{3}$ & $\widehat{\Omega}_{4}$ \\
\hline$\widehat{\Omega}_{5}$ & $\hat{\Omega}_{6}$ & $\widehat{\Omega}_{7}$ & $\widehat{\Omega}_{8}$ \\
\hline$\hat{\Omega}_{9}$ & $\hat{\Omega}_{10}$ & $\hat{\Omega}_{11}$ & $\widehat{\Omega}_{12}$ \\
\hline$\widehat{\Omega}_{13}$ & $\hat{\Omega}_{14}$ & $\widehat{\Omega}_{15}$ & $\widehat{\Omega}_{16}$ \\
\hline
\end{tabular}

Figure 3.

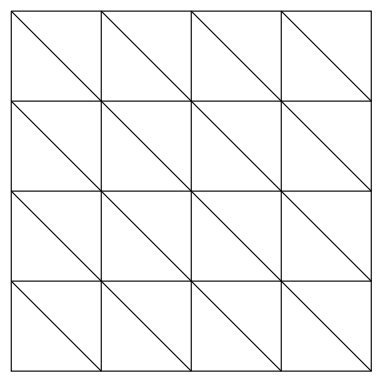

Figure 4.

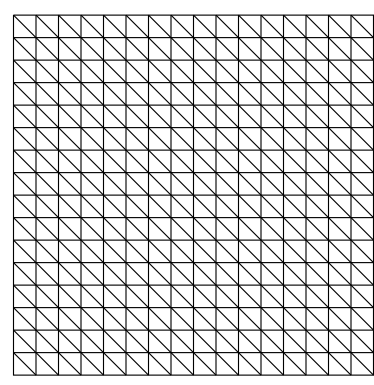

Figure 5.

Let $V_{h} \subseteq V$ be the finite element space associated with $\mathcal{T}_{h}$. The discretization of (1.3) is:

Find $u_{h} \in V_{h}$ such that

$$
a\left(u_{h}, v\right)=\int_{\Omega} f v d x \quad \forall v \in V_{h}
$$

Let $A_{h}: V_{h} \longrightarrow V_{h}^{\prime}$ be the linear operator from $V_{h}$ to its dual space defined by

$$
\left\langle A_{h} v_{1}, v_{2}\right\rangle=a\left(v_{1}, v_{2}\right) \quad \forall v_{1}, v_{2} \in V_{h}
$$

where $\langle\cdot, \cdot\rangle$ is the canonical bilinear form between a vector space and its dual. The operator $A_{h}$ is symmetric positive definite (SPD) in the sense that $\left\langle A_{h} v_{1}, v_{2}\right\rangle=\left\langle A_{h} v_{2}, v_{1}\right\rangle$ for all $v_{1}, v_{2} \in V_{h}$ and $\left\langle A_{h} v, v\right\rangle>0$ for $0 \neq v \in V_{h}$. Note that if $f_{h} \in V_{h}^{\prime}$ is defined by $\left\langle f_{h}, v\right\rangle=\int_{\Omega} f v d x$ for all $v \in V_{h}$, then (1.4) can be written as $A_{h} u_{h}=f_{h}$.

The two-level additive Schwarz preconditioner (cf. [8], [21] and the references therein) for $A_{h}$ is constructed as follows. Let $\widehat{\Omega}_{j}$ be enlarged in all directions by the amount $\delta=\ell h$ $\left(\ell \in \mathbb{N}\right.$ ) and $\Omega_{j}$ be the intersection of this enlarged square with $\Omega$ (cf. Figure 6 ). 


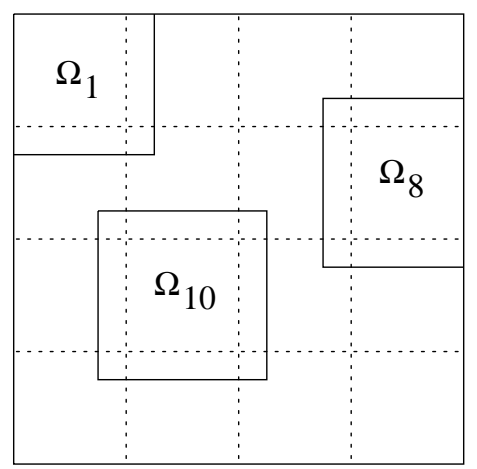

Figure 6.

We define $V_{H} \subseteq V$ to be the finite element space associated with $\mathcal{T}_{H}$, and $V_{j}$ to be the subspace of $V_{h}$ whose members vanish identically outside $\Omega_{j}$, for $1 \leq j \leq J$. The SPD operators $A_{H}: V_{H} \longrightarrow V_{H}^{\prime}$ and $A_{j}: V_{j} \longrightarrow V_{j}^{\prime}$ are defined by

$$
\begin{aligned}
\left\langle A_{H} v_{1}, v_{2}\right\rangle=a\left(v_{1}, v_{2}\right) & \forall v_{1}, v_{2} \in V_{H}, \\
\left\langle A_{j} v_{1}, v_{2}\right\rangle=a\left(v_{1}, v_{2}\right) & \forall v_{1}, v_{2} \in V_{j} .
\end{aligned}
$$

The operators $I_{H}: V_{H} \longrightarrow V_{h}$ and $I_{j}: V_{j} \longrightarrow V_{h}$ are just natural injections, and we denote by $I_{H}^{t}: V_{h}^{\prime} \longrightarrow V_{H}^{\prime}$ and $I_{j}^{t}: V_{h}^{\prime} \longrightarrow V_{j}^{\prime}$ their transposes with respect to the canonical bilinear forms, i.e.,

$$
\begin{aligned}
& \left\langle I_{H}^{t} \alpha, v\right\rangle=\left\langle\alpha, I_{H} v\right\rangle \quad \forall \alpha \in V_{h}^{\prime}, v \in V_{H}, \\
& \left\langle I_{j}^{t} \alpha, v\right\rangle=\left\langle\alpha, I_{j} v\right\rangle \quad \forall \alpha \in V_{h}^{\prime}, v \in V_{j} .
\end{aligned}
$$

The two-level additive Schwarz preconditioner $B: V_{h}^{\prime} \longrightarrow V_{h}$ is defined by

$$
B=I_{H} A_{H}^{-1} I_{H}^{t}+\sum_{j=1}^{J} I_{j} A_{j}^{-1} I_{j}^{t} .
$$

It is easy to check that $B A_{h}: V_{h} \longrightarrow V_{h}$ is SPD with respect to the bilinear form $\langle A \cdot, \cdot\rangle=a(\cdot, \cdot)$. It is known (cf. [13], [23]) that for second order problems

$$
\kappa\left(B A_{h}\right) \leq C\left(1+\frac{H}{\delta}\right)
$$

and for fourth order problems (cf. [4], [3])

$$
\kappa\left(B A_{h}\right) \leq C\left(1+\frac{H}{\delta}\right)^{3}
$$

where the (generic) constant $C$ in (1.11) and (1.12) is independent of $h, H, J$ and $\delta$. 
In this paper we will show that for $\delta=h$ (minimal overlap) the following estimate holds for the second order model problem

$$
\kappa\left(B A_{h}\right) \geq c\left(\frac{H}{h}\right)
$$

while the estimate

$$
\kappa\left(B A_{h}\right) \geq c\left(\frac{H}{h}\right)^{3}
$$

holds for the fourth order model problem, where the (generic) positive constant $c$ is independent of $h, H$ and $J$.

Hence, the known upper bounds are sharp in the case of a small overlap for both second and fourth order problems. We note that the sharpness of (1.11) has already been remarked upon in [13].

The rest of the paper is organized as follows. Section 2 contains some lemmas that are needed in the subsequent sections. We prove the lower bound (1.13) for the second order model problem in Section 3 and the lower bound (1.14) for the fourth order model problem in Section 4.

\section{Some Lemmas}

First we state an abstract result for additive Schwarz preconditioners. Let $\mathscr{V}$ and $\mathscr{W}_{j}$, $0 \leq j \leq J$, be finite dimensional vector spaces, and $\mathscr{A}: \mathscr{V} \longrightarrow \mathscr{V}^{\prime}$ and $\mathscr{B}_{j}: \mathscr{W}_{j} \longrightarrow \mathscr{W}^{\prime}$ be linear SPD operators. Let the vectors spaces be connected by the linear operators $\mathscr{I}_{j}: \mathscr{W}_{j} \longrightarrow \mathscr{V}$. Then the additive Schwarz preconditioner $\mathscr{B}: \mathscr{V}^{\prime} \longrightarrow \mathscr{V}$ is defined by

$$
\mathscr{B}=\sum_{j=0}^{J} \mathscr{I}_{j} \mathscr{B}_{j}^{-1} \mathscr{I}_{j}^{t},
$$

where $\mathscr{I}_{j}^{t}: \mathscr{V}^{\prime} \longrightarrow \mathscr{W}^{\prime}$ is the transpose of $\mathscr{I}_{j}$ with respect to the canonical bilinear forms. We have the following lemma (cf. [17], [19], [20], [12], [24], [14]) on the eigenvalues of $\mathscr{B} \mathscr{A}$.

Lemma 2.1. The operator $\mathscr{B} \mathscr{A}$ is symmetric positive semi-definite with respect to $\langle\mathscr{A} \cdot, \cdot\rangle$. The minimum eigenvalue $\lambda_{\min }(\mathscr{B} \mathscr{A})$ and the maximum eigenvalue $\lambda_{\max }(\mathscr{B} \mathscr{A})$ of $\mathscr{B} \mathscr{A}$ have the following characterizations:

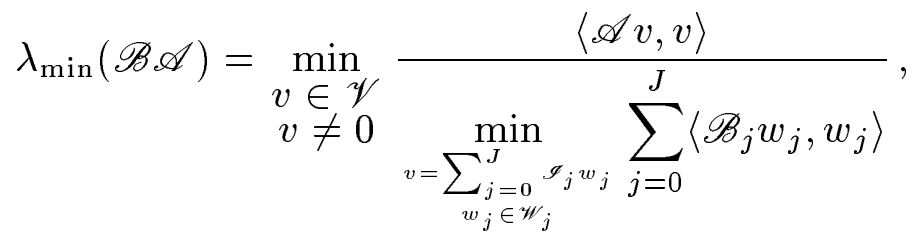

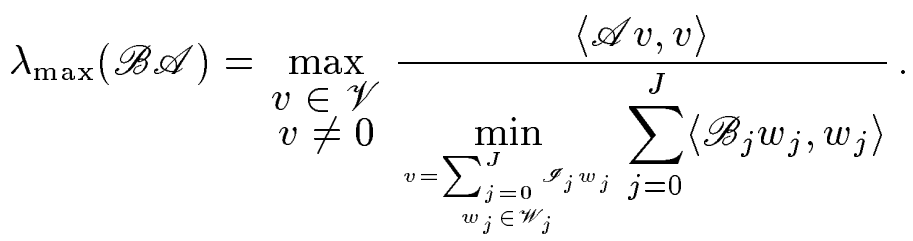


Next we state three lemmas concerning discrete norms and semi-norms for finite element spaces. They can all be easily proved by straight-forward calculations and standard scaling arguments. The Sobolev semi-norms in these lemmas are defined by

$$
|v|_{H^{\ell}(G)}=\left(\int_{G} \sum_{|\alpha|=\ell}\left(\partial_{x}^{\alpha} v\right)^{2} d x\right)^{1 / 2},
$$

where $G$ is an open subset of $\mathbb{R}^{n}, \partial_{x}^{\alpha}=\partial_{x_{1}}^{\alpha_{1}} \partial_{x_{2}}^{\alpha_{2}} \cdots \partial_{x_{n}}^{\alpha_{n}}$ and $|\alpha|=\alpha_{1}+\cdots+\alpha_{n}$.

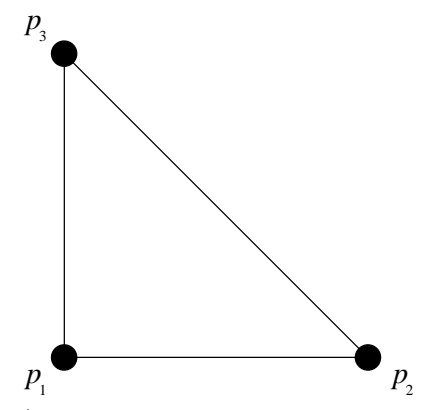

Figure 7.

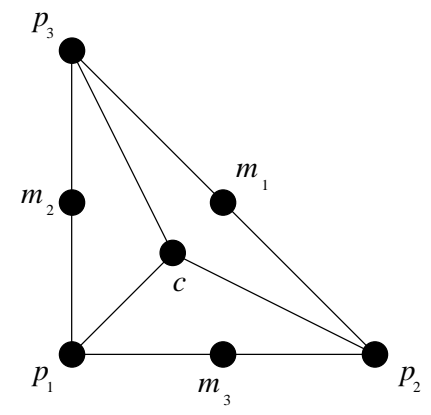

Figure 8 .

Lemma 2.2. Let $v\left(x_{1}, x_{2}\right)$ be a linear polynomial on an isosceles right-angled triangle $T$ with vertices $p_{1}, p_{2}$ and $p_{3}$ (cf. Figure 7 ). Then there exists a positive constant $C$ independent of diam $T$ and $v$ such that

$$
\sum_{j=2,3}\left[v\left(p_{1}\right)-v\left(p_{j}\right)\right]^{2} \leq C|v|_{H^{1}(T)}^{2} .
$$

Lemma 2.3. Let $v\left(x_{1}, x_{2}\right)$ be a $C^{1}$ function on an isosceles right-angled triangle $T$ such that $v$ is piecewise cubic with respect to the triangulation formed by the vertices $p_{i}(1 \leq i \leq 3)$ and the centroid $c$ of $T$ (cf. Figure 8). Let $m_{i}, 1 \leq i \leq 3$, be the midpoints of the three sides of $T$. Then there exists a positive constant $C$ independent of $\operatorname{diam} T$ and $v$ such that

$$
\begin{gathered}
\sum_{i=1,2} \sum_{j=2,3}\left[v_{x_{i}}\left(p_{1}\right)-v_{x_{i}}\left(p_{j}\right)\right]^{2}+\left(\frac{v\left(p_{1}\right)-v\left(p_{2}\right)}{\left|p_{1} p_{2}\right|}-\frac{\partial v}{\partial n}\left(m_{2}\right)\right)^{2} \\
+\left(\frac{v\left(p_{1}\right)-v\left(p_{3}\right)}{\left|p_{1} p_{3}\right|}-\frac{\partial v}{\partial n}\left(m_{3}\right)\right)^{2} \leq C|v|_{H^{2}(T)}^{2}
\end{gathered}
$$

where $\partial v / \partial n$ denotes the normal derivative of $v$ in the direction of the outer normal.

Lemma 2.4. Let $\mathcal{I}$ be an interval with endpoints $p_{1}$ and $p_{2}$. Let $P_{1}(\mathcal{I}), P_{3}(\mathcal{I})$ be respectively the space of linear and cubic polynomials defined on $\mathcal{I}$. Then there exist positive constants $C_{1}$ and $C_{2}$ independent of $|\mathcal{I}|$ such that

$$
\begin{array}{ll}
\|v\|_{L_{2}(\mathcal{I})}^{2} \leq C_{1}|\mathcal{I}| \sum_{i=1,2} v^{2}\left(p_{i}\right) & \forall v \in P_{1}(\mathcal{I}), \\
\|v\|_{L_{2}(\mathcal{I})}^{2} \leq C_{3}|\mathcal{I}| \sum_{i=1,2}\left[v^{2}\left(p_{i}\right)+|\mathcal{I}|^{2}\left(v^{\prime}\right)^{2}\left(p_{i}\right)\right] & \forall v \in P_{3}(\mathcal{I}) .
\end{array}
$$




\section{The Second Order Case}

In this section we consider the preconditioner $B$ (cf. (1.10)) for the second order model problem, where $V=H_{0}^{1}(\Omega), a(\cdot, \cdot)$ is defined by $(1.1)$, and the $P_{1}$ conforming finite element is used. The overlap $\delta$ is taken to be $h$, i.e., we consider the case of minimal overlap.

In order to avoid the proliferation of constants, we will henceforth use the notation $A \lesssim B$ (or $B \gtrsim A$ ) to represent the statement that $A \leq$ constant $\times B$, where the constant is independent of $h, H, J$ and the variables in $A$ and $B$. The notation $A \approx B$ means that $A \lesssim B$ and $A \gtrsim B$.

First we apply Lemma 2.1 to obtain a lower bound for $\lambda_{\max }\left(B A_{h}\right)$. In this context we have $\mathscr{V}=V_{h}, \mathscr{W}_{0}=V_{H}, \mathscr{W}_{j}=V_{j}$ for $1 \leq j \leq J, \mathscr{A}=A_{h}, \mathscr{B}_{0}=A_{H}, \mathscr{B}_{j}=A_{j}$ for $1 \leq j \leq J, \mathscr{I}_{0}=I_{H}$, and $\mathscr{I}_{j}=I_{j}$ for $1 \leq j \leq J$.

Lemma 3.1. The following estimate holds:

$$
\lambda_{\max }\left(B A_{h}\right) \geq 1 .
$$

Proof. Let $0 \neq v_{*} \in V_{1}$. We have a trivial decomposition of $v_{*}: v_{*}=v_{H}+\sum_{j=1}^{J} v_{j}$, where $0=v_{H}=v_{2}=\cdots=v_{J}$ and $v_{1}=v_{*}$. It follows from (1.5)-(1.7) and (ii) of Lemma 2.1 that

$$
\lambda_{\max }\left(B A_{h}\right) \geq a\left(v_{*}, v_{*}\right) /\left(\min _{\substack{v_{*}=v_{H}+\sum_{j=1}^{J} v_{H} \in V_{H}, v_{j} \in V_{j} \\ v_{H}}}\left[a\left(v_{H}, v_{H}\right)+\sum_{j=1}^{J} a\left(v_{j}, v_{j}\right)\right]\right) \geq \frac{a\left(v_{*}, v_{*}\right)}{a\left(v_{*}, v_{*}\right)}=1 .
$$

By (1.5)-(1.7) and (i) of Lemma 2.1, in order to show that $\lambda_{\min }\left(B A_{h}\right) \lesssim(h / H)$, it suffices to find one function $v_{\dagger} \in V_{h}$ such that

$$
a\left(v_{\dagger}, v_{\dagger}\right) \lesssim\left(\frac{h}{H}\right) \min _{\substack{v_{\dagger}=v_{H}+\sum_{j=1}^{J} v_{H} \in v_{H}, v_{j} \in V_{j} \\ v_{j}}}\left[a\left(v_{H}, v_{H}\right)+\sum_{j=1}^{J} a\left(v_{j}, v_{j}\right)\right] .
$$

We will construct $v_{\dagger}$ as one of the discrete harmonic functions associated with the nonoverlapping decomposition $\widehat{\Omega}_{1}, \ldots, \widehat{\Omega}_{J}$ (cf. Figure 3 ).

Let $\Gamma=\left(\bigcup_{j=1}^{J} \partial \widehat{\Omega}_{j}\right) \backslash \partial \Omega$ be the skeleton of the nonoverlapping decomposition. The subspace $V_{h}(\Omega \backslash \Gamma)$ of $V_{h}$ is defined by

$$
V_{h}(\Omega \backslash \Gamma)=\left\{v \in V_{h}: v \text { vanishes on } \Gamma\right\} .
$$

The subspace $V_{h}(\Gamma)$ of $V_{h}$ is the $a(\cdot, \cdot)$-orthogonal complement of $V_{h}(\Omega \backslash \Gamma)$, i.e.,

$$
V_{h}(\Gamma)=\left\{v \in V_{h}: a(v, w)=0 \quad \forall w \in V_{h}(\Omega \backslash \Gamma)\right\} .
$$

The functions in $V_{h}(\Gamma)$ are known as discrete harmonic functions and they are completely determined by their nodal values along $\Gamma$. The property of discrete harmonic functions that we will use is stated in the following lemma, the proof of which can be found in [2] and [22]. 
Lemma 3.2. The following estimate holds:

$$
|v|_{H^{1}\left(\widehat{\Omega}_{j}\right)} \approx|v|_{H^{1 / 2}\left(\partial \widehat{\Omega}_{j}\right)} \quad \text { for } 1 \leq j \leq J \quad \text { and } \quad \forall v \in V_{h}(\Gamma)
$$

The fractional order Sobolev semi-norm $|\cdot|_{H^{1 / 2}\left(\partial \widehat{\Omega}_{j}\right)}$ in Lemma 3.2 is defined by

$$
|v|_{H^{1 / 2}\left(\partial \widehat{\Omega}_{j}\right)}^{2}=\int_{\partial \widehat{\Omega}_{j}} \int_{\partial \widehat{\Omega}_{j}} \frac{|v(x)-v(y)|^{2}}{|x-y|^{2}} d s(x) d s(y)
$$

where $d s$ denotes the differential of the arc length.

Let $\mathrm{P}_{1} \mathrm{P}_{2}$ be the common boundary of two subdomains $\widehat{\Omega}_{j_{1}}$ and $\widehat{\Omega}_{j_{2}}$ which is parallel to the $x_{1}$-axis, and $\mathrm{Q}_{1}, \mathrm{Q}_{2}$ be two points on $\mathrm{P}_{1} \mathrm{P}_{2}$ such that $\left|\mathrm{P}_{1} \mathrm{Q}_{1}\right|=\left|\mathrm{P}_{2} \mathrm{Q}_{2}\right|=H / 4$ (cf. Figure 9).

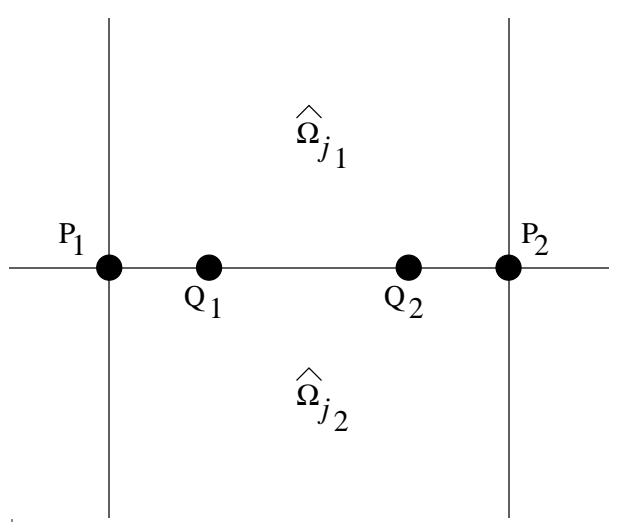

Figure 9 .

The restriction to $\Gamma$ of the function $v_{\dagger} \in V_{h}(\Gamma)$ that we are going to construct will vanish outside the line segment $\mathrm{Q}_{1} \mathrm{Q}_{2}$. Lemma 3.2 and a simple calculation shows that for such functions the following lemma holds.

Lemma 3.3. Suppose that $v \in V_{h}(\Gamma)$ and $\left.v\right|_{\Gamma}$ vanishes outside $\mathrm{Q}_{1} \mathrm{Q}_{2}$. Then we have

$$
|v|_{H^{1}(\Omega)} \approx|v|_{H^{1 / 2}\left(\mathrm{P}_{1} \mathrm{P}_{2}\right)},
$$

where

$$
|v|_{H^{1 / 2}\left(\mathrm{P}_{1} \mathrm{P}_{2}\right)}^{2}=\int_{\mathrm{P}_{1} \mathrm{P}_{2}} \int_{\mathrm{P}_{1} \mathrm{P}_{2}} \frac{|v(x)-v(y)|^{2}}{|x-y|^{2}} d x_{1} d y_{1} .
$$

In view of Lemma 3.3, we can focus our construction to the reference interval $I=[0,1]$. Let $\mathcal{T}_{\rho}$ be a dyadic subdivision of $I$ with mesh size $\rho$ and $\mathcal{L}_{\rho}(I)$ be the space of continuous piecewise linear functions on $I$ associated with $\mathcal{T}_{\rho}$.

Since the dimension of the subspace $\left\{w \in \mathcal{L}_{1 / 8}(I): w=0\right.$ outside $\left.(1 / 4,3 / 4)\right\}$ of $\mathcal{L}_{1 / 8}(I)$ is three, there exists a nontrivial function $\hat{g}$ with the following properties: 
(i) $\hat{g} \in \mathcal{L}_{1 / 8}(I)$

(ii) $\hat{g}=0$ outside $(1 / 4,3 / 4)$,

(iii) $\int_{1 / 4}^{3 / 4} \hat{g}(x) d x=\int_{1 / 4}^{3 / 4} x \hat{g}(x) d x=0$.

We denote by $\alpha$ the constant $\left(|\hat{g}|_{H^{1 / 2}(I)}^{2} /|\hat{g}|_{L_{2}(I)}^{2}\right)$, which is of course independent of $h, H$ and $J$.

The next lemma follows from the construction on $I$ above and a scaling argument.

Lemma 3.4. There exists a continuous function $g$ defined on the line segment $\mathrm{P}_{1} \mathrm{P}_{2}$ (cf. Figure 9) which is piecewise linear with respect to the dyadic subdivision induced by $\mathcal{T}_{h}$, for any $h \leq(H / 8)$, and which has the following properties:

$$
g \text { vanishes outside the line segment } \mathrm{Q}_{1} \mathrm{Q}_{2} \text { (cf. Figure 9), }
$$

$$
\int_{\mathrm{Q}_{1} \mathrm{Q}_{2}} g(x) v(x) d x_{1}=0 \quad \text { for any } v \text { which is a linear polynomial on } \mathrm{P}_{1} \mathrm{P}_{2} \text {, }
$$$$
\frac{|g|_{H^{1 / 2}\left(\mathrm{P}_{1} \mathrm{P}_{2}\right)}^{2}}{\|g\|_{L_{2}\left(\mathrm{Q}_{1} \mathrm{Q}_{2}\right)}^{2}}=\frac{\alpha}{H} .
$$

For $(h / H) \leq(1 / 8)$, we can now define $v_{\dagger} \in V_{h}(\Gamma)$ to be the discrete harmonic function which vanishes everywhere on $\Gamma$ except the segment $\mathrm{P}_{1} \mathrm{P}_{2}$, where it is identical to the function $g$ in Lemma 3.4. It follows from (1.1), Lemma 3.3, (3.4) and (3.6) that

$$
a\left(v_{\dagger}, v_{\dagger}\right) \lesssim \frac{1}{H}\left(v_{\dagger}, v_{\dagger}\right)_{L_{2}\left(\mathrm{Q}_{1} \mathrm{Q}_{2}\right)} .
$$

Given any decomposition

$$
v_{\dagger}=v_{H}+\sum_{j=1}^{J} v_{j}
$$

where $v_{H} \in V_{H}$ and $v_{j} \in V_{j}$ for $1 \leq j \leq J$, we have, since the overlap is minimal,

$$
\left.\left(v_{\dagger}-v_{H}\right)\right|_{\mathrm{Q}_{1} \mathrm{Q}_{2}}=\left.v_{j_{1}}\right|_{\mathrm{Q}_{1} \mathrm{Q}_{2}}+\left.v_{j_{2}}\right|_{\mathrm{Q}_{1} \mathrm{Q}_{2}} .
$$

It follows from (3.5) and (3.9) that

$$
\left(v_{\dagger}, v_{\dagger}\right)_{L_{2}\left(\mathrm{Q}_{1} \mathrm{Q}_{2}\right)} \leq\left(v_{\dagger}-v_{H}, v_{\dagger}-v_{H}\right)_{L_{2}\left(\mathrm{Q}_{1} \mathrm{Q}_{2}\right)} \lesssim\left\|v_{j_{1}}\right\|_{L_{2}\left(\mathrm{Q}_{1} \mathrm{Q}_{2}\right)}^{2}+\left\|v_{j_{2}}\right\|_{L_{2}\left(\mathrm{Q}_{1} \mathrm{Q}_{2}\right)}^{2} .
$$

Let $p_{\ell}, 1 \leq \ell \leq L$, be the dyadic subdivision points on $\mathrm{Q}_{1} \mathrm{Q}_{2}$ induced by $\mathcal{T}_{h}$. Part (i) of Lemma 2.4 implies that

$$
\left\|v_{j_{1}}\right\|_{L_{2}\left(\mathrm{Q}_{1} \mathrm{Q}_{2}\right)}^{2}+\left\|v_{j_{2}}\right\|_{L_{2}\left(\mathrm{Q}_{1} \mathrm{Q}_{2}\right)}^{2} \lesssim h \sum_{\ell=1}^{L}\left[v_{j_{1}}^{2}\left(p_{\ell}\right)+v_{j_{2}}^{2}\left(p_{\ell}\right)\right] .
$$

Since the overlap is minimal, each $p_{\ell}$ belongs to a triangle $T_{\ell} \in \mathcal{T}_{h}$ where $v_{j_{2}}$ vanishes at all the vertices except $p_{\ell}$. These triangles appear as the shaded triangles in Figure 10. 


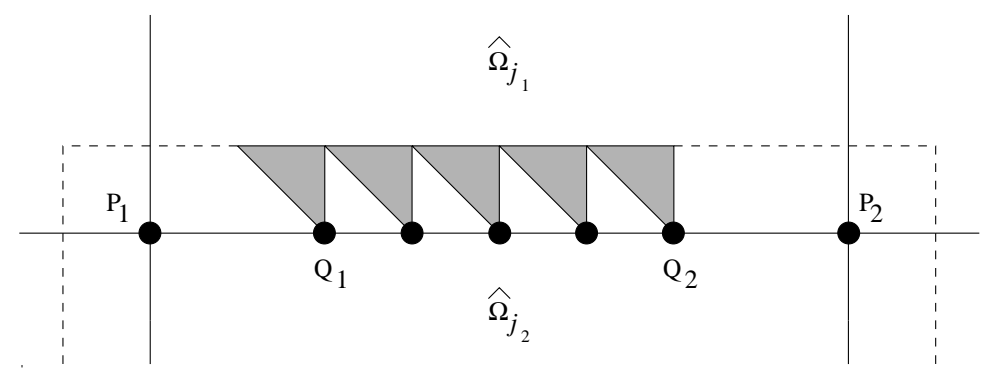

Figure 10.

Hence, by Lemma 2.2, we have

$$
\sum_{\ell=1}^{L} v_{j_{2}}^{2}\left(p_{\ell}\right) \lesssim \sum_{\ell=1}^{L}\left|v_{j_{2}}\right|_{H^{1}\left(T_{\ell}\right)}^{2} \leq\left|v_{j_{2}}\right|_{H^{1}(\Omega)}^{2}=a\left(v_{j_{2}}, v_{j_{2}}\right)
$$

Similarly we have

$$
\sum_{\ell=1}^{L} v_{j_{1}}^{2}\left(p_{\ell}\right) \lesssim a\left(v_{j_{1}}, v_{j_{1}}\right)
$$

Combining (3.7) and (3.10)-(3.13) we find

$$
a\left(v_{\dagger}, v_{\dagger}\right) \lesssim\left(\frac{h}{H}\right)\left[a\left(v_{j_{1}}, v_{j_{1}}\right)+a\left(v_{j_{2}}, v_{j_{2}}\right)\right]
$$

for any decomposition of $v_{\dagger}$ given by (3.8), which implies (3.2) and hence the next lemma.

Lemma 3.5. For $(h / H) \leq(1 / 8)$ we have

$$
\lambda_{\min }\left(B A_{h}\right) \lesssim\left(\frac{h}{H}\right) .
$$

Finally we can establish the estimate (1.13).

Theorem 3.6. There exists a positive constant $c$ independent of $h, H$ and $J$ such that

$$
\kappa\left(B A_{h}\right) \geq c\left(\frac{H}{h}\right)
$$

holds for the second order model problem in the case of minimal overlap.

Proof. For $(h / H) \leq(1 / 8)$ the estimate follows (3.1) and (3.14). On the other hand, the estimate follows from the trivial estimate $1 \leq \kappa\left(B A_{h}\right)$ when $(h / H) \geq(1 / 8)$.

Remark 3.7. It is easy to see that Theorem 3.6 can be applied to many other elements and that the estimate

$$
\kappa\left(B A_{h}\right) \geq c\left(\frac{H}{\delta}\right)
$$

is valid under the condition that $(\delta / h)$ is bounded. Note also that $(3.15)$ can be extended to the second order model problem on the unit cube $(0,1)^{3}$. 
Remark 3.8. The estimate (3.15) can also be extended to nonconforming finite elements.

Remark 3.9. When $H$ is fixed and the overlap $\delta \approx h$, we have $\kappa\left(B A_{h}\right) \approx h^{-1}$, which is also the estimate for the condition number of the Schur complement in nonoverlapping domain decomposition algorithms for second order problems (cf. [1], [18], [5]).

\section{The Fourth Order Case}

We consider in this section the fourth order model problem where $V=H_{0}^{2}(\Omega), a(\cdot, \cdot)$ is defined by (1.2) and the Hsieh-Clough-Tocher macro element is used. The overlap $\delta$ is again taken to be $h$.

The first lemma is established by the same argument in the proof of Lemma 3.1.

Lemma 4.1. The following estimate holds:

$$
\lambda_{\max }\left(B A_{h}\right) \geq 1 .
$$

By (i) of Lemma 2.1, in order to show that $\lambda_{\min }\left(B A_{h}\right) \lesssim(h / H)^{3}$, it suffices to find one function $v_{\dagger} \in V_{h}$ such that

$$
a\left(v_{\dagger}, v_{\dagger}\right) \lesssim\left(\frac{h}{H}\right)^{3} \min _{\substack{v_{\dagger}=v_{H}+\sum_{j=1}^{J} v_{H} \in V_{H}, v_{j} \in V_{j} \\ v_{j}}}\left[a\left(v_{H}, v_{H}\right)+\sum_{j=1}^{J} a\left(v_{j}, v_{j}\right)\right]
$$

We will construct $v_{\dagger}$ as one of the discrete biharmonic functions associated with the nonoverlapping decomposition $\widehat{\Omega}_{1}, \ldots, \widehat{\Omega}_{J}$ (cf. Figure 3 ).

Let $\Gamma=\left(\bigcup_{j=1}^{J} \partial \widehat{\Omega}_{j}\right) \backslash \partial \Omega$ be the skeleton. The subspace $V_{h}(\Omega \backslash \Gamma)$ is defined by

$$
V_{h}(\Omega \backslash \Gamma)=\left\{v \in V_{h}: v \text { vanishes to the first order on } \Gamma\right\} .
$$

The subspace $V_{h}(\Gamma)$ is then defined as in (3.3). The functions in $V_{h}(\Gamma)$ are known as discrete biharmonic functions and they are completely determined by their nodal values (i.e., derivatives up to order one at the vertices and normal derivatives at the midpoints (cf. Figure 2)) along $\Gamma$. The proof of the following property of discrete biharmonic functions can be found in [15], [16] and [7].

Lemma 4.2. The following estimate holds:

$$
|v|_{H^{2}\left(\widehat{\Omega}_{j}\right)} \approx \sum_{i=1,2}\left|v_{x_{i}}\right|_{H^{1 / 2}\left(\partial \widehat{\Omega}_{j}\right)} \quad \text { for } 1 \leq j \leq J \quad \text { and } \quad \forall v \in V_{h}(\Gamma)
$$

Using Lemma 4.2 and referring to Figure 9, we obtain the following lemma by a simple calculation. 
Lemma 4.3. Suppose that $v \in V_{h}(\Gamma)$ and $\left.v\right|_{\Gamma}$ vanishes to the first order outside $\mathrm{Q}_{1} \mathrm{Q}_{2}$. Then we have

$$
|v|_{H^{2}(\Omega)} \approx \sum_{i=1,2}\left|v_{x_{i}}\right|_{H^{1 / 2}\left(\mathrm{P}_{1} \mathrm{P}_{2}\right)} .
$$

Note that the restriction of $v \in V_{h}$ to $\mathrm{P}_{1} \mathrm{P}_{2}$ is a $C^{1}$ function which is piecewise cubic.

In view of Lemma 4.3 we can again focus our construction to the reference interval $I=[0,1]$. Let $\mathcal{T}_{\rho}$ be a dyadic subdivision of $I$ with mesh size $\rho$ and $\mathcal{C}_{\rho}(I)$ be the space of $C^{1}$ functions which are piecewise cubic with respect to $\mathcal{T}_{\rho}$.

Since the dimension of the subspace $\left\{w \in \mathcal{C}_{1 / 8}(I): w=0\right.$ outside $\left.(1 / 4,3 / 4)\right\}$ of $\mathcal{C}_{1 / 8}(I)$ is six, there exists a nontrivial $g \in \mathcal{C}_{1 / 8}(I)$ with the following properties:

(i) $\hat{g} \in \mathcal{L}_{1 / 8}(I)$

(ii) $\hat{g}=0$ outside $(1 / 4,3 / 4)$,

(iii) $\int_{1 / 4}^{3 / 4} x^{k} \hat{g}(x) d x=0$ for $k=0,1,2,3$.

We denote by $\beta$ the constant $\left(\left|\hat{g}^{\prime}\right|_{H^{1 / 2}(I)}^{2} /\|\hat{g}\|_{L_{2}(I)}^{2}\right)$, which is independent of $h, H$ and $J$.

The following lemma is obtained by a scaling argument.

Lemma 4.4. There exists a $C^{1}$ function $g$ defined on the line segment $\mathrm{P}_{1} \mathrm{P}_{2}$ (cf. Figure 9 ) which is piecewise cubic with respect to the dyadic subdivision induced by $\mathcal{T}_{h}$, for any $h \leq(H / 8)$, and which has the following properties:

$$
g \text { vanishes outside the line segment } \mathrm{Q}_{1} \mathrm{Q}_{2} \text { (cf. Figure 9), }
$$

$$
\begin{aligned}
& \int_{\mathrm{Q}_{1} \mathrm{Q}_{2}} g(x) v(x) d x_{1}=0 \quad \text { for any } v \text { which is a cubic polynomial on } \mathrm{P}_{1} \mathrm{P}_{2}, \\
& \frac{\left|g_{x_{1}}\right|_{H^{1 / 2}\left(\mathrm{P}_{1} \mathrm{P}_{2}\right)}^{2}}{\|g\|_{L_{2}\left(\mathrm{Q}_{1} \mathrm{Q}_{2}\right)}^{2}}=\left(\frac{\beta}{H}\right)^{3}
\end{aligned}
$$

For $(h / H) \leq(1 / 8)$ we define $v_{\dagger} \in V_{h}(\Gamma)$ to be the discrete biharmonic function which vanishes to the first order everywhere on $\Gamma$ except the segment $\mathrm{P}_{1} \mathrm{P}_{2}$. On $\mathrm{P}_{1} \mathrm{P}_{2}$ it satisfies the following conditions:

$$
\begin{aligned}
\left.v_{\dagger}\right|_{\mathrm{P}_{1} \mathrm{P}_{2}} & =g, \\
\left.\left(v_{\dagger}\right)_{x_{2}}\right|_{\mathrm{P}_{1} \mathrm{P}_{2}} & =0,
\end{aligned}
$$

where $g$ is the function in Lemma 4.4. It follows from (1.2), Lemma 4.3, (4.3) and (4.5)(4.7) that

$$
a\left(v_{\dagger}, v_{\dagger}\right) \lesssim\left(\frac{1}{H}\right)^{3}\left(v_{\dagger}, v_{\dagger}\right)_{L_{2}\left(\mathrm{Q}_{1} \mathrm{Q}_{2}\right)}
$$


Given any decomposition of $v_{\dagger}$ defined by (3.8), we have, by (4.4),

$$
\left(v_{\dagger}, v_{\dagger}\right)_{L_{2}\left(\mathrm{Q}_{1} \mathrm{Q}_{2}\right)} \leq\left(v_{\dagger}-v_{H}, v_{\dagger}-v_{H}\right)_{L_{2}\left(\mathrm{Q}_{1} \mathrm{Q}_{2}\right)} \lesssim\left\|v_{j_{1}}\right\|_{L_{2}\left(\mathrm{Q}_{1} \mathrm{Q}_{2}\right)}^{2}+\left\|v_{j_{2}}\right\|_{L_{2}\left(\mathrm{Q}_{1} \mathrm{Q}_{2}\right)}^{2} .
$$

Let $p_{\ell}, 1 \leq \ell \leq L$ be the dyadic subdivision points on $\mathrm{Q}_{1} \mathrm{Q}_{2}$ induced by $\mathcal{T}_{h}$. It follows from (ii) of Lemma 2.4 that

$$
\begin{aligned}
\left\|v_{j_{1}}\right\|_{L_{2}\left(\mathrm{Q}_{1} \mathrm{Q}_{2}\right)}^{2}+\left\|v_{j_{2}}\right\|_{L_{2}\left(\mathrm{Q}_{1} \mathrm{Q}_{2}\right)}^{2} \lesssim h \sum_{\ell=1}^{L} & {\left[v_{j_{1}}^{2}\left(p_{\ell}\right)+v_{j_{2}}^{2}\left(p_{\ell}\right)\right] } \\
& +h^{3} \sum_{\ell=1}^{L}\left[\left(v_{j_{1}}\right)_{x_{1}}^{2}\left(p_{\ell}\right)+\left(v_{j_{2}}\right)_{x_{1}}^{2}\left(p_{\ell}\right)\right] .
\end{aligned}
$$

Since the overlap is minimal, each $p_{\ell}$ belongs to a triangle $T_{\ell} \in \mathcal{T}_{h}$ where all the nodal values of $v_{j_{2}}$ vanish on the side opposite to $p_{\ell}$ (cf. Figure 10). Hence, by Lemma 2.3, we have

$$
\begin{gathered}
\sum_{\ell=1}^{L} v_{j_{2}}^{2}\left(p_{\ell}\right) \lesssim h^{2} \sum_{\ell=1}^{L}\left|v_{j_{2}}\right|_{H^{2}\left(T_{\ell}\right)}^{2} \leq h^{2}\left|v_{j_{2}}\right|_{H^{2}(\Omega)}^{2}=h^{2} a\left(v_{j_{2}}, v_{j_{2}}\right), \\
\sum_{\ell=1}^{L}\left(v_{j_{2}}\right)_{x_{1}}^{2}\left(p_{\ell}\right) \lesssim \sum_{\ell=1}^{L}\left|v_{j_{2}}\right|_{H^{2}\left(T_{\ell}\right)}^{2} \leq\left|v_{j_{2}}\right|_{H^{2}(\Omega)}^{2}=a\left(v_{j_{2}}, v_{j_{2}}\right),
\end{gathered}
$$

and similarly,

$$
\begin{gathered}
\sum_{\ell=1}^{L} v_{j_{1}}^{2}\left(p_{\ell}\right) \lesssim h^{2} a\left(v_{j_{1}}, v_{j_{1}}\right), \\
\sum_{\ell=1}^{L}\left(v_{j_{1}}\right)_{x_{1}}^{2}\left(p_{\ell}\right) \lesssim a\left(v_{j_{1}}, v_{j_{1}}\right) .
\end{gathered}
$$

Combining (4.8)-(4.14) we find

$$
a\left(v_{\dagger}, v_{\dagger}\right) \lesssim\left(\frac{h}{H}\right)^{3}\left[a\left(v_{j_{1}}, v_{j_{1}}\right)+a\left(v_{j_{2}}, v_{j_{2}}\right)\right]
$$

which implies (4.2) and hence the following lemma.

Lemma 4.5. For $(h / H) \leq(1 / 8)$ we have

$$
\lambda_{\min }\left(B A_{h}\right) \lesssim\left(\frac{h}{H}\right)^{3}
$$


Using (4.1) and (4.15) and the argument in the proof of Theorem 3.6 we obtain the following theorem.

Theorem 4.6. There exists a positive constant $c$ independent of $h, H$ and $J$ such that

$$
\kappa\left(B A_{h}\right) \geq c\left(\frac{H}{h}\right)^{3}
$$

holds for the fourth order model problem in the case of minimal overlap.

Remark 4.7. It is easy to see that Theorem 4.6 can be applied to many other elements and that the estimate

$$
\kappa\left(B A_{h}\right) \geq c\left(\frac{H}{\delta}\right)^{3}
$$

is valid under the condition that $(\delta / h)$ is bounded. The estimate (4.16) can also be extended to nonconforming finite elements. When $H$ is fixed and the overlap $\delta \approx h$, we have $\kappa\left(B A_{h}\right) \approx h^{-3}$, which is also the estimate for the condition number of the Schur complement in nonoverlapping domain decomposition algorithms for fourth order problems (cf. [9], [5]).

Acknowledgment The research in this paper began while the author was visiting the Institute for Mathematics and its Applications at the University of Minnesota. She would like to thank the IMA for their support and hospitality.

\section{References}

1. P.E. Bjørstad and O.B. Widlund, Iterative methods for the solution of elliptic problems on regions partitioned into substructures, SIAM J. Numer. Anal. 23 (1986), 1097-1120.

2. J.H. Bramble, J.E. Pasciak and A.H. Schatz, The construction of preconditioners for elliptic problems by substructuring,I, Math. Comp. 47 (1986), 103-104.

3. S.C. Brenner, A two-level additive Schwarz preconditioner for nonconforming plate elements, Numer. Math. 72 (1996), 419-447.

4 . A two-level additive Schwarz preconditioner for macro-element approximations of the plate bending problem, Houston J. Math. 21 (1995), 823-844.

5. tion, IMI Research Report 97:05, University of South Carolina, 1997.

6. S.C. Brenner and L.R. Scott, The Mathematical Theory of Finite Element Methods, Springer-Verlag, New York, 1994.

7. S.C. Brenner and L.-Y. Sung, Balancing domain decomposition for nonconforming plate elements, IMI Research Report 97:04, University of South Carolina, 1997.

8. T.F. Chan and T.P. Mathew, Domain decomposition algorithms, Acta Numerica (1994), $61-143$. 
9. T.F. Chan, W. E and J. Sun, Domain decomposition interface preconditioners for fourth-order elliptic problems, Appl. Numer. Math. 8 (1991), 317-331.

10. P.G. Ciarlet, The Finite Element Method for Elliptic Problems, North Holland, Amsterdam, 1978.

11. R.W. Clough and J.L. Tocher, Finite element stiffness matrices for for analysis of plates in bending, Proceedings of the Conference on Matrix Methods in Structural Mechanics (1965), Wright Patterson A.F.B., Ohio.

12. M. Dryja and O.B. Widlund, Some domain decomposition algorithms for elliptic problems, in Iterative Methods for Large Linear Systems (L. Hayes and D. Kincaid, eds), 273-291, Academic Press, New York, 1989.

13.

Domain decomposition algorithms with small overlap, SIAM J. Sci. Comp. 15 (1994), 604-620.

14. M. Griebel and P. Oswald, On the abstract theory of additive and multiplicative Schwarz algorithms, Numer. Math. 70 (1995), 163-180.

15. P. Le Tallec, J. Mandel and M. Vidrascu, Balancing domain decomposition for plates, in Domain Decomposition Methods in Scientific and Engineering Computing, Contemporary Mathematics 180 (D.E. Keyes et al, eds), American Mathematical Society, Providence, 1994, 515-524.

16. P. Le Tallec, J. Mandel and M. Vidrascu, A Neumann-Neumann domain decomposition algorithm for solving plate and shell problems, preprint (1997).

17. P. Lions, On the Schwarz alternating method. I, in First International Symposium on Domain Decomposition Methods for Partial Differential Equations (R. Glowinski et al, eds), 1-42, SIAM, Philadelphia, 1988.

18. L. Mansfield, On the conjugate gradient solution of the Schur complement system obtained from domain decomposition, SIAM J. Numer. Anal. 27 (1990), 1612-1620.

19. S.V. Nepomnyaschikh, On the application of the bordering method to the mixed boundary value problem for elliptic equations and on mesh norms in $W^{1 / 2}(S)$, Soviet J. Numer. Anal. Math. Modelling 4 (1989), 493-506.

20. , Fictitious components and subdomain alternating methods, Soviet J. Numer. Anal. Math. Modelling 5 (1990), 53-68.

21. B. Smith, P. Bjørstad and W. Gropp, Domain Decomposition, Cambridge University Press, Cambridge, 1996.

22. O.B. Widlund, An extension theorem for finite element spaces with three applications, in Numerical Techniques in Continuum Mechanics (W. Hackbusch and K. Witsch, eds), 110-122, Friedr. Vieweg und Sohn, Braunschweig/Wiesbaden, 1987.

23. Some Schwarz methods for symmetric and nonsymmetric elliptic problems, in Fifth International Symposium on Domain Decomposition Methods for Partial Differential Equations (D.E. Keyes et al, eds), 19-36, SIAM, Philadelphia, 1991.

24. X. Zhang, Studies in Domain Decomposition: Multi-level Methods and the Biharmonic Dirichlet Problem, Dissertation, Courant Institute, 1991. 